\title{
Android Recipes
}

\section{A Problem-Solution Approach for Android 5.0 \\ Fourth Edition}

Dave Smith 


\section{Android Recipes: A Problem-Solution Approach for Android 5.0}

\section{Copyright (C) 2015 by Dave Smith}

This work is subject to copyright. All rights are reserved by the Publisher, whether the whole or part of the material is concerned, specifically the rights of translation, reprinting, reuse of illustrations, recitation, broadcasting, reproduction on microfilms or in any other physical way, and transmission or information storage and retrieval, electronic adaptation, computer software, or by similar or dissimilar methodology now known or hereafter developed. Exempted from this legal reservation are brief excerpts in connection with reviews or scholarly analysis or material supplied specifically for the purpose of being entered and executed on a computer system, for exclusive use by the purchaser of the work. Duplication of this publication or parts thereof is permitted only under the provisions of the Copyright Law of the Publisher's location, in its current version, and permission for use must always be obtained from Springer. Permissions for use may be obtained through RightsLink at the Copyright Clearance Center. Violations are liable to prosecution under the respective Copyright Law.

ISBN-13 (pbk): 978-1-4842-0476-4

ISBN-13 (electronic): 978-1-4842-0475-7

Trademarked names, logos, and images may appear in this book. Rather than use a trademark symbol with every occurrence of a trademarked name, logo, or image we use the names, logos, and images only in an editorial fashion and to the benefit of the trademark owner, with no intention of infringement of the trademark.

The images of the Android Robot (01/Android Robot) are reproduced from work created and shared by Google and used according to terms described in the Creative Commons 3.0 Attribution License. Android and all Android and Google-based marks are trademarks or registered trademarks of Google Inc. in the United States and other countries. Apress Media LLC is not affiliated with Google Inc., and this book was written without endorsement from Google Inc.

The use in this publication of trade names, trademarks, service marks, and similar terms, even if they are not identified as such, is not to be taken as an expression of opinion as to whether or not they are subject to proprietary rights.

While the advice and information in this book are believed to be true and accurate at the date of publication, neither the authors nor the editors nor the publisher can accept any legal responsibility for any errors or omissions that may be made. The publisher makes no warranty, express or implied, with respect to the material contained herein.

Managing Director: Welmoed Spahr

Lead Editor: Steve Anglin

Development Editor: Matthew Moodie

Technical Reviewer: Paul Trebilcox-Ruiz

Editorial Board: Steve Anglin, Louise Corrigan, Jonathan Gennick, Robert Hutchinson, Michelle Lowman, James Markham, Matthew Moodie, Jeff Olson, Jeffrey Pepper, Douglas Pundick, Ben Renow-Clarke, Gwenan Spearing, Steve Weiss

Coordinating Editor: Mark Powers

Copy Editor: Sharon Wilkey

Compositor: SPi Global

Indexer: SPi Global

Artist: SPi Global

Cover Designer: Anna Ishchenko

Distributed to the book trade worldwide by Springer Science + Business Media New York, 233 Spring Street, 6th Floor, New York, NY 10013. Phone 1-800-SPRINGER, fax (201) 348-4505, e-mail orders-ny@springer-sbm.com, or visit wWw. springeronline. com. Apress Media, LLC is a California LLC and the sole member (owner) is Springer Science + Business Media Finance Inc (SSBM Finance Inc). SSBM Finance Inc is a Delaware corporation.

For information on translations, please e-mail rights@apress.com, or visitwwW.apress.com.

Apress and friends of ED books may be purchased in bulk for academic, corporate, or promotional use. eBook versions and licenses are also available for most titles. For more information, reference our Special Bulk Sales-eBook Licensing web page at wWw. apress. com/bulk-sales.

Any source code or other supplementary material referenced by the author in this text is available to readers at WWw. apress.com/9781484204764. For detailed information about how to locate your book's source code, go to WWW. apress.com/source-code/. 


\section{Contents at a Glance}

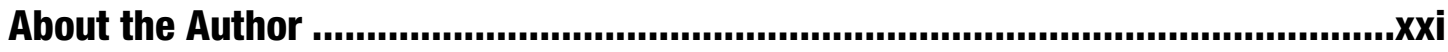

About the Technical Reviewer ..........................................................................xxiii

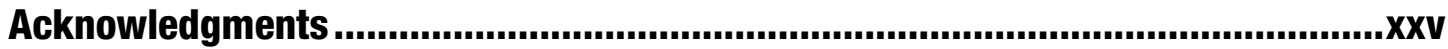

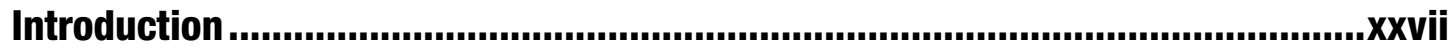

Chapter 1: Layouts and Views ................................................................... 1

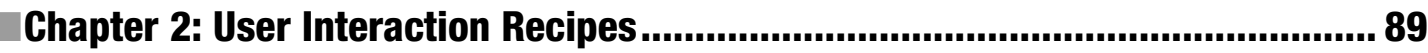

Chapter 3: Communications and Networking ................................................... 199

Chapter 4: Interacting with Device Hardware and Media................................ 289

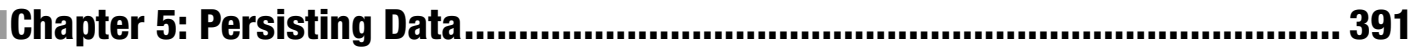

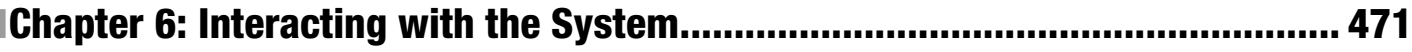

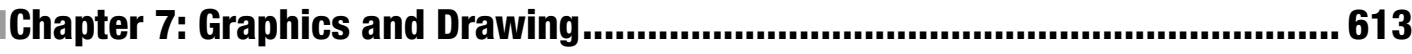

Chapter 8: Working with Android NDK and RenderScript................................... 689

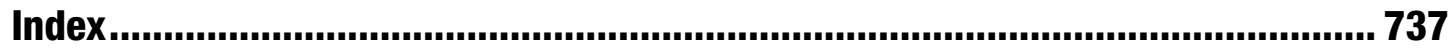





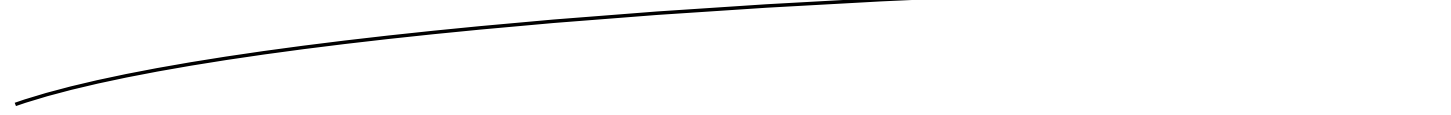

\section{Contents}

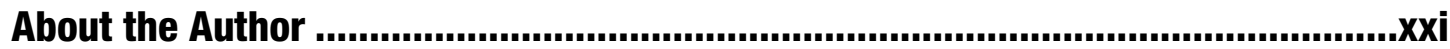

About the Technical Reviewer ...................................................................iii

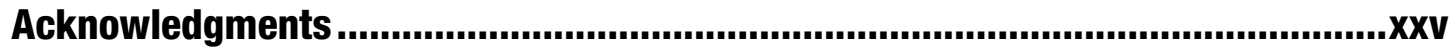

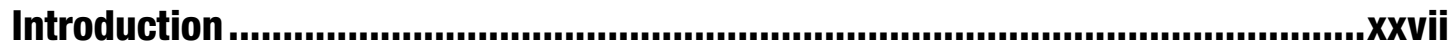

Chapter 1: Layouts and Views ........................................................................ 1

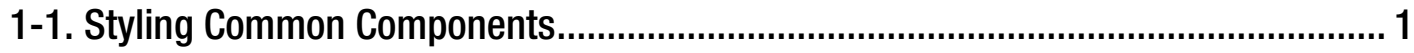

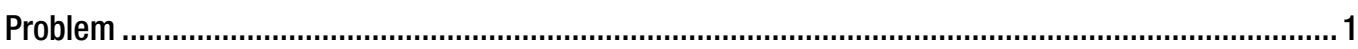

Solution

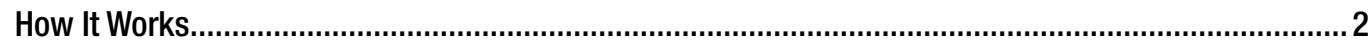

1-2. Toggling System UI Elements........................................................................... 11

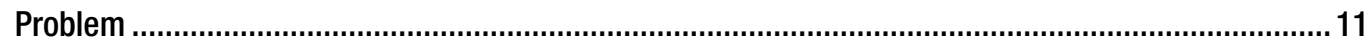

Solution

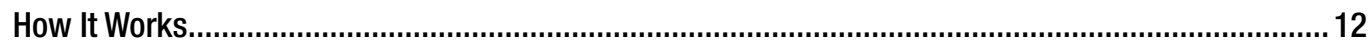

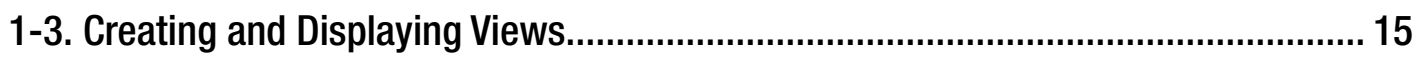

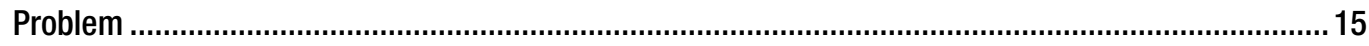

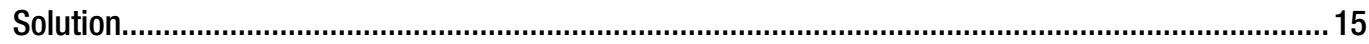

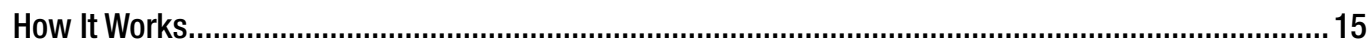




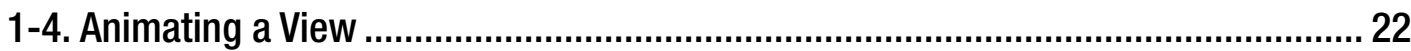

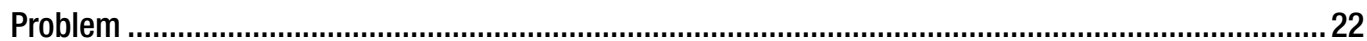

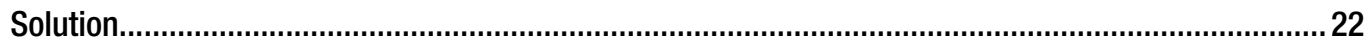

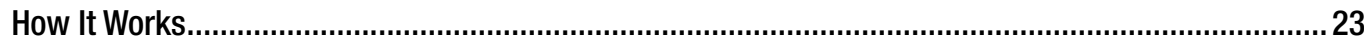

1-5. Animating Layout Changes ............................................................................... 28

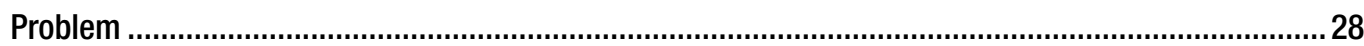

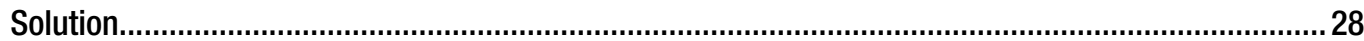

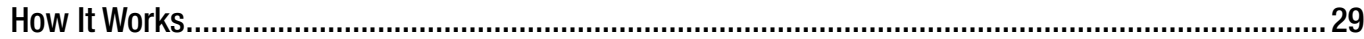

1-6. Implementing Situation-Specific Layouts........................................................... 32

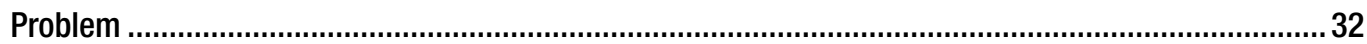

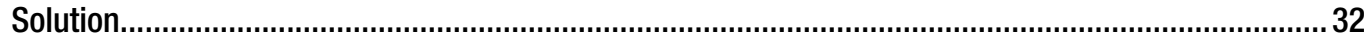

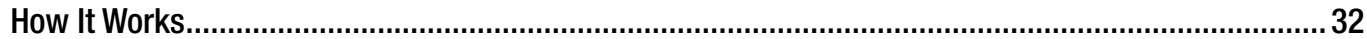

1-7. Customizing AdapterView Empty Views............................................................ 42

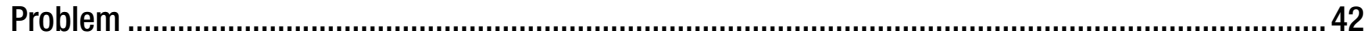

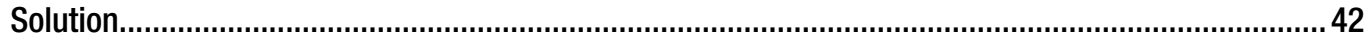

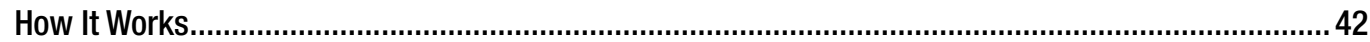

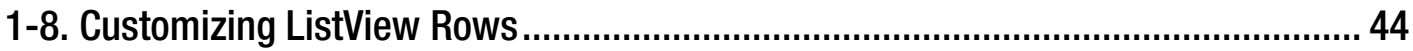

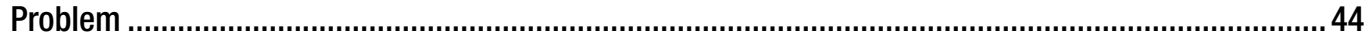

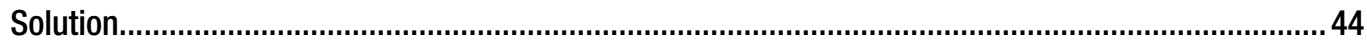

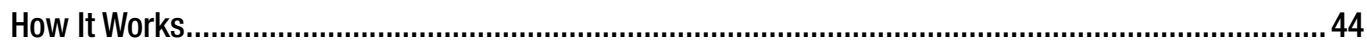

1-9. Making ListView Section Headers ...................................................................... 48

Problem

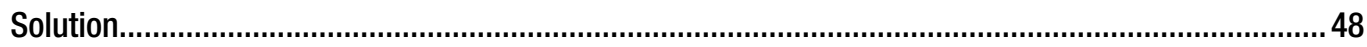

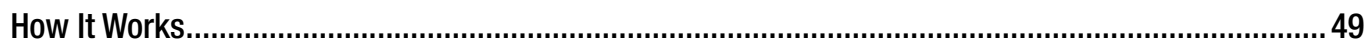

1-10. Creating Compound Controls ......................................................................... 56

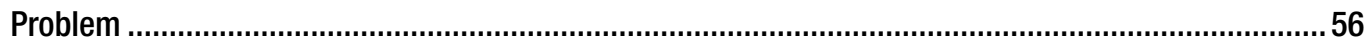

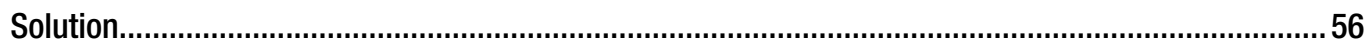

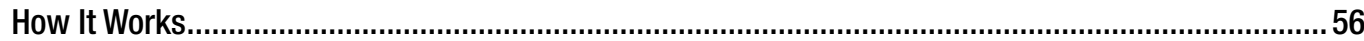

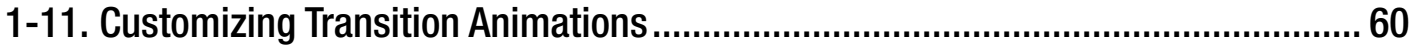

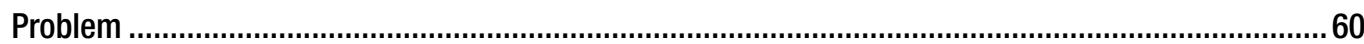

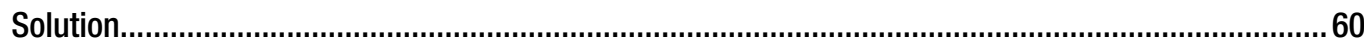

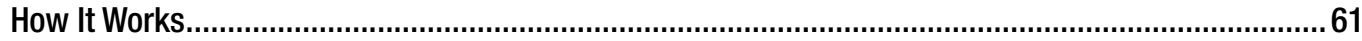




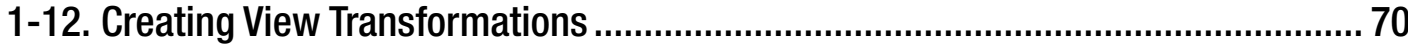

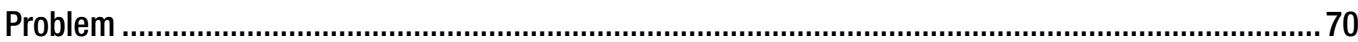

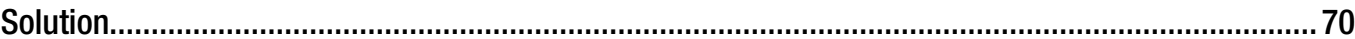

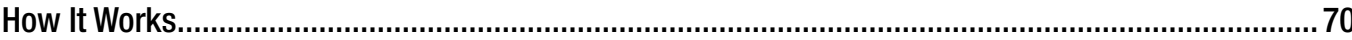

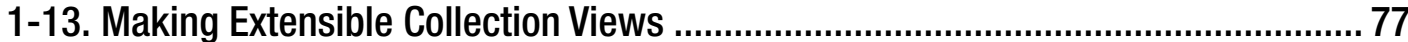

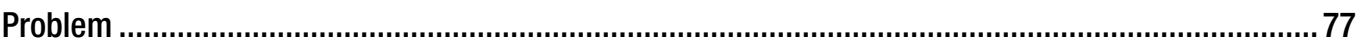

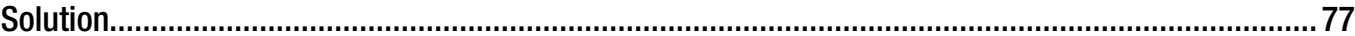

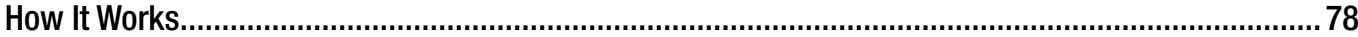

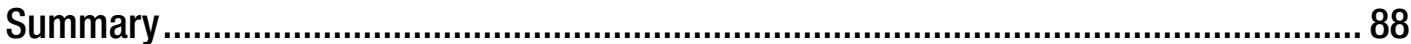

Chapter 2: User Interaction Recipes................................................................. 89

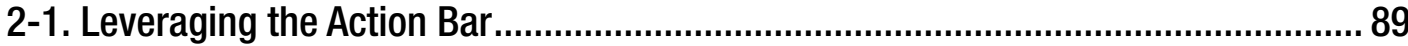

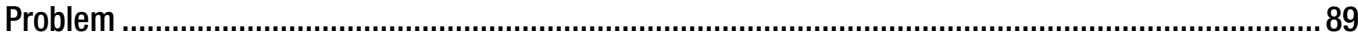

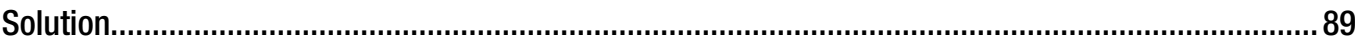

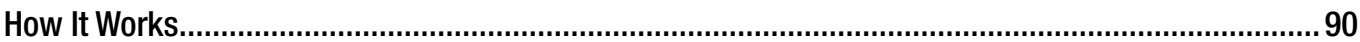

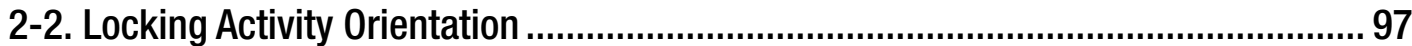

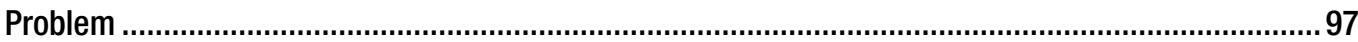

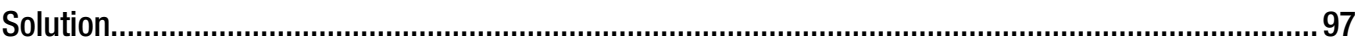

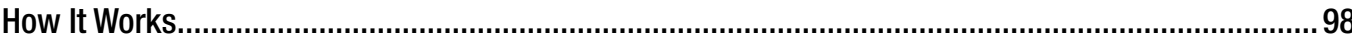

2-3. Performing Dynamic Orientation Locking ............................................................ 98

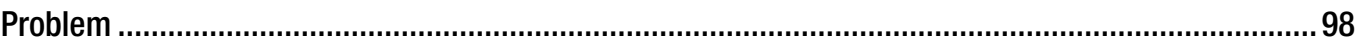

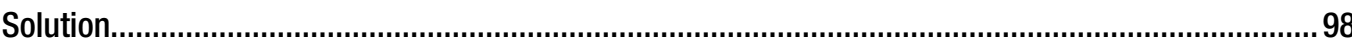

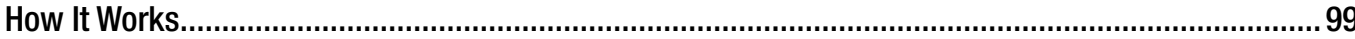

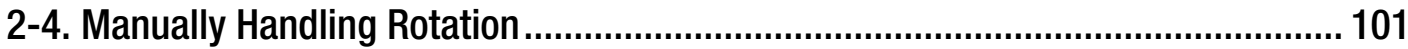

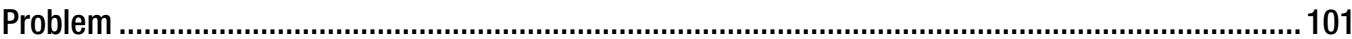

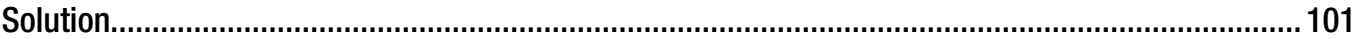

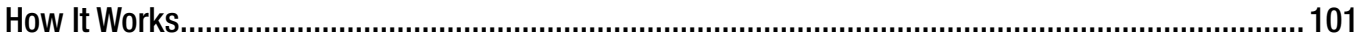

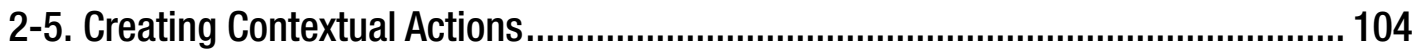

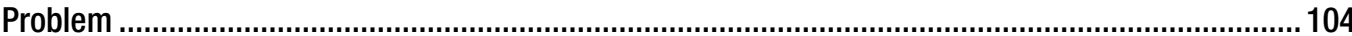

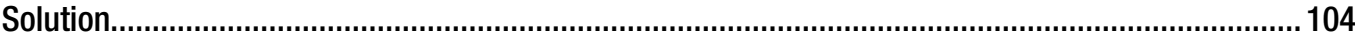

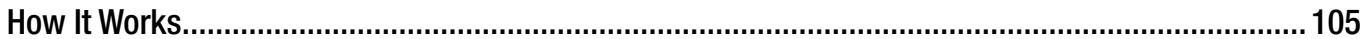


2-6. Displaying a User Dialog Box.............................................................................. 110

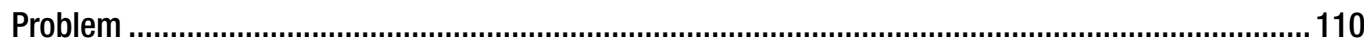

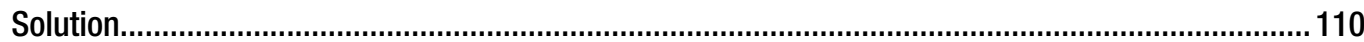

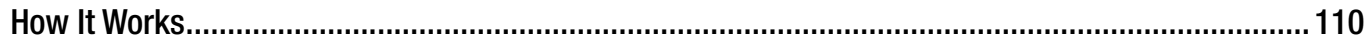

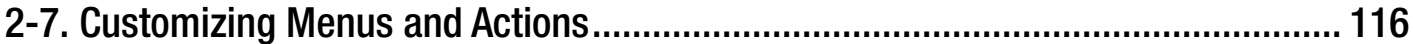

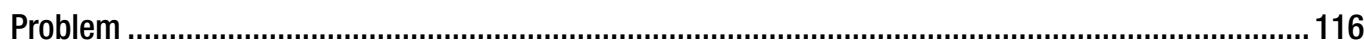

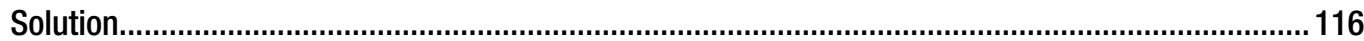

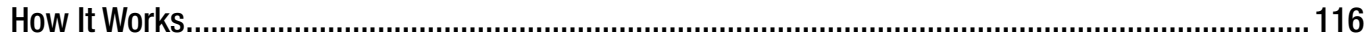

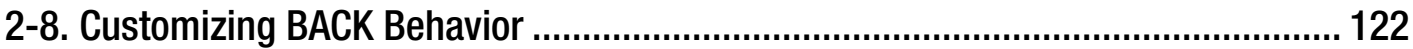

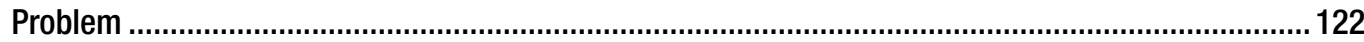

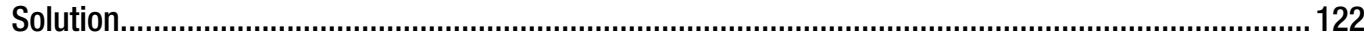

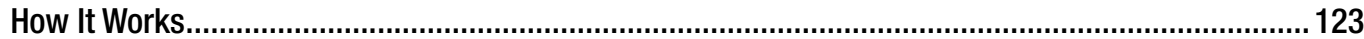

2-9. Emulating the HOME Button.......................................................................... 126

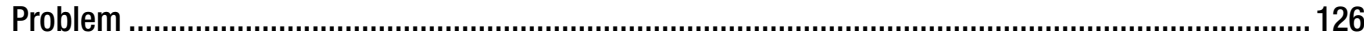

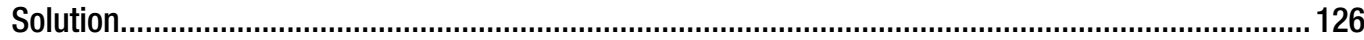

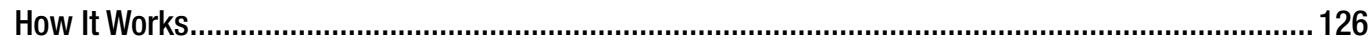

2-10. Monitoring TextView Changes...................................................................... 127

Problem

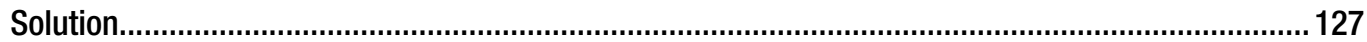

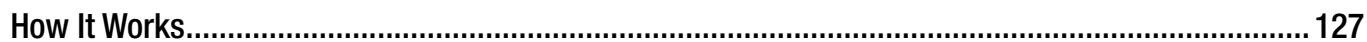

2-11. Customizing Keyboard Actions ................................................................... 130

Problem

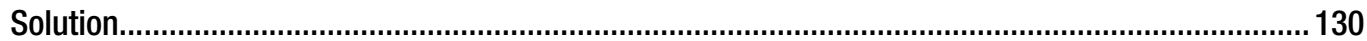

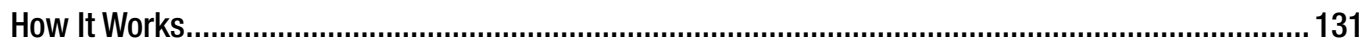

2-12. Dismissing the Soft Keyboard................................................................... 134

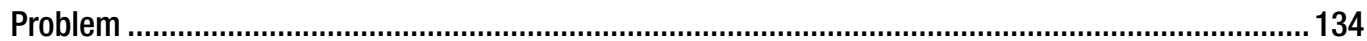

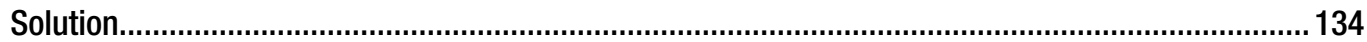

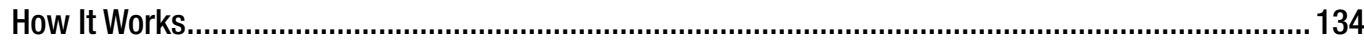

2-13. Handling Complex Touch Events .................................................................. 134

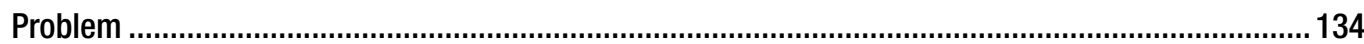

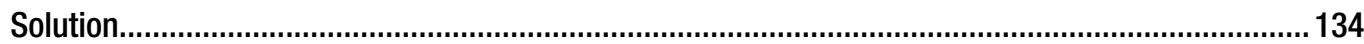

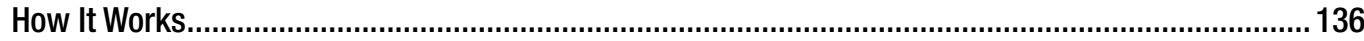




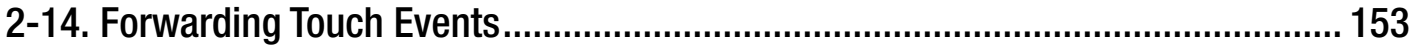

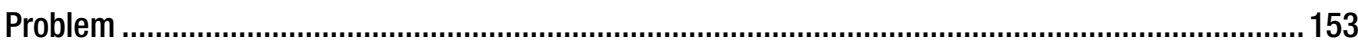

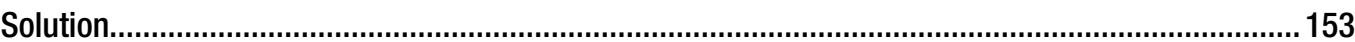

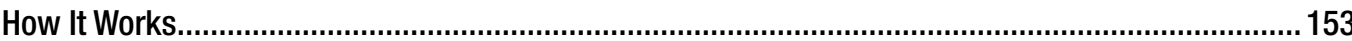

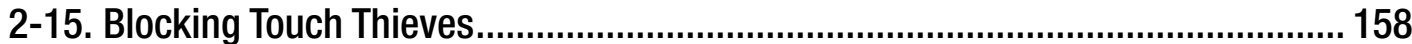

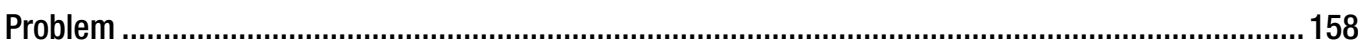

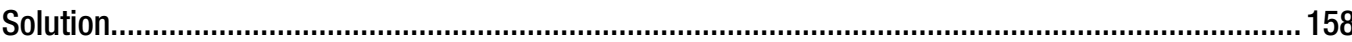

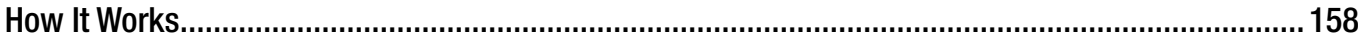

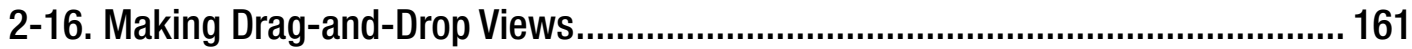

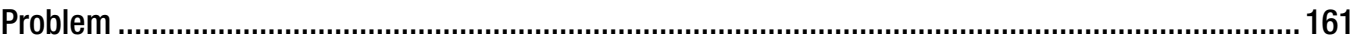

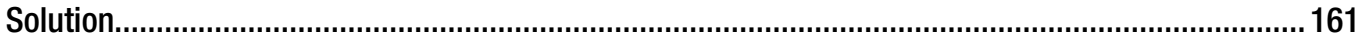

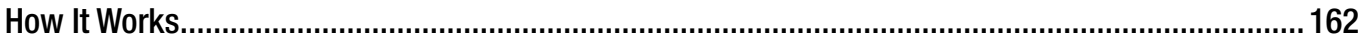

2-17. Building a Navigation Drawer ...................................................................... 169

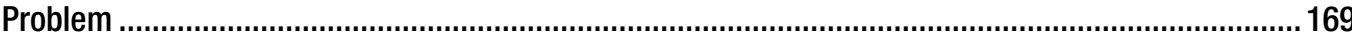

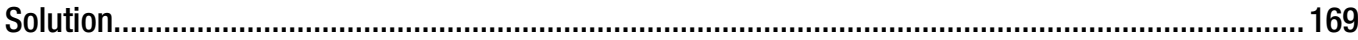

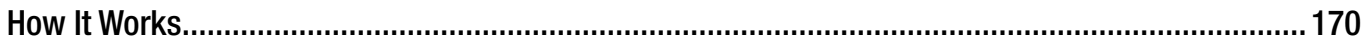

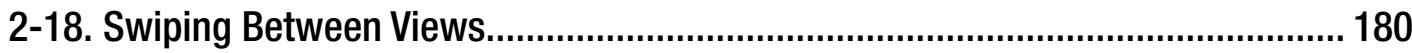

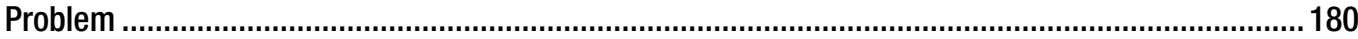

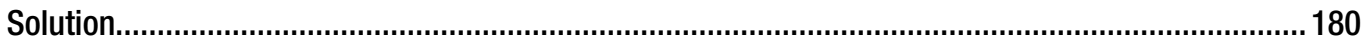

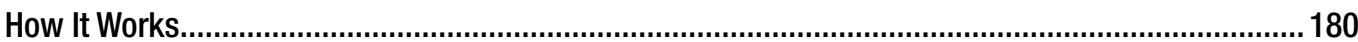

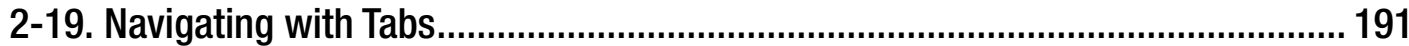

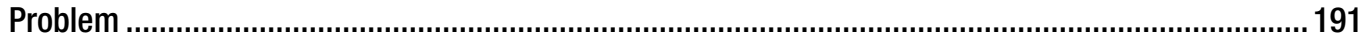

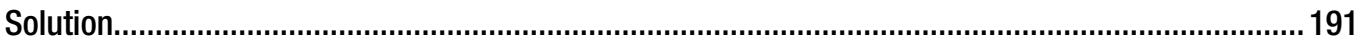

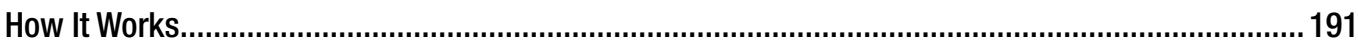

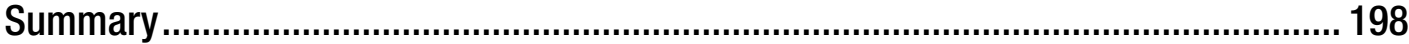

Chapter 3: Communications and Networking ................................................. 199

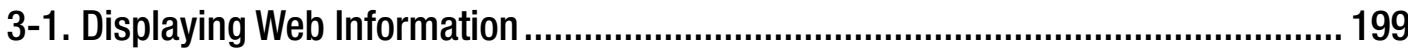

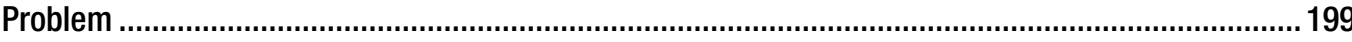

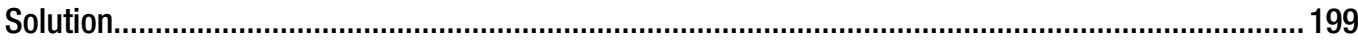

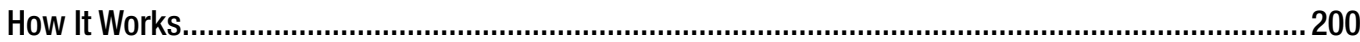




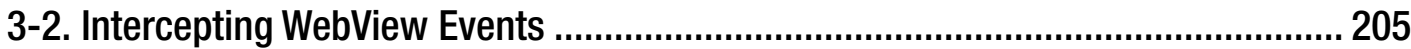

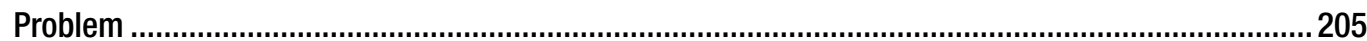

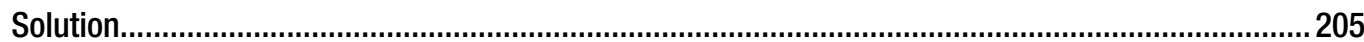

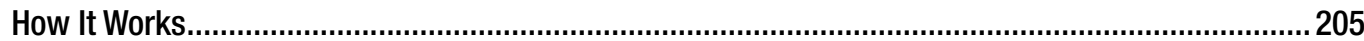

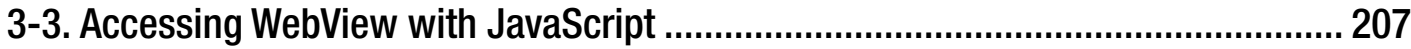

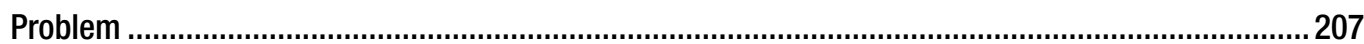

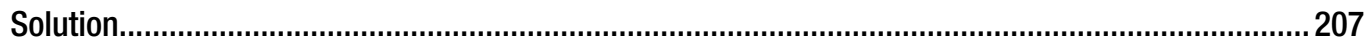

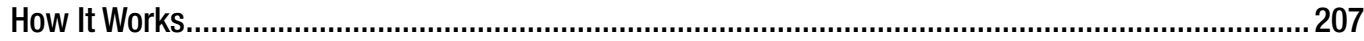

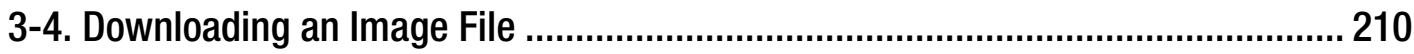

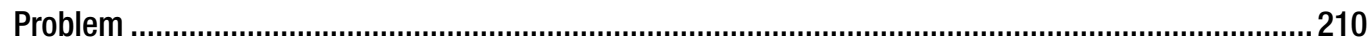

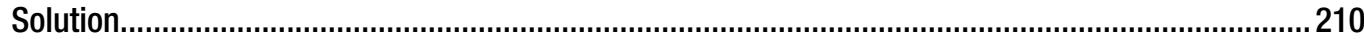

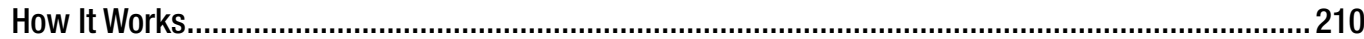

3-5. Downloading Completely in the Background................................................. 213

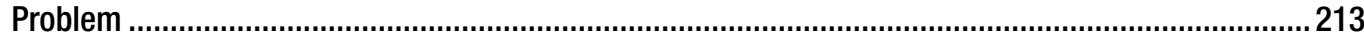

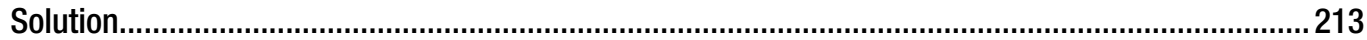

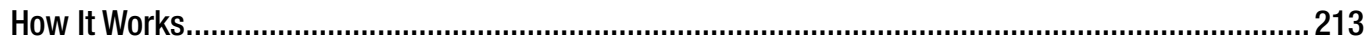

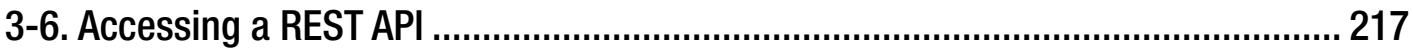

Problem

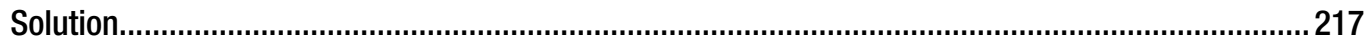

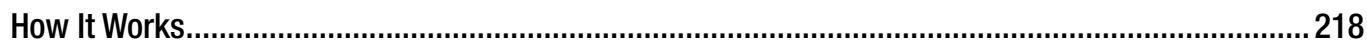

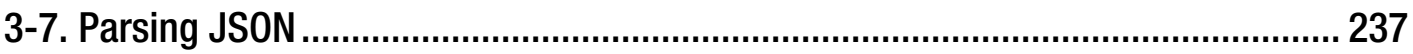

Problem

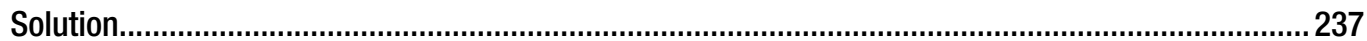

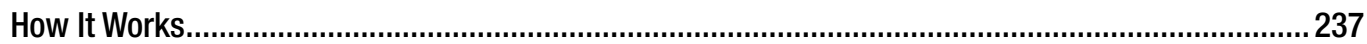

3-8. Parsing XML

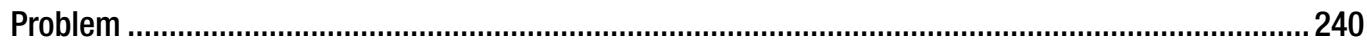

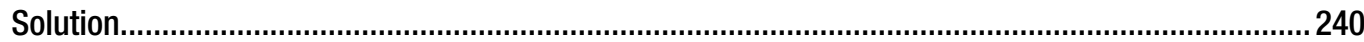

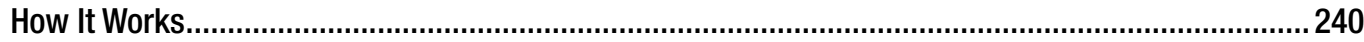


3-9. Receiving SMS

Problem 251

Solution 251

How It Works .251

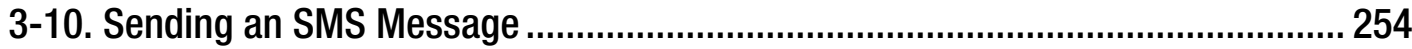

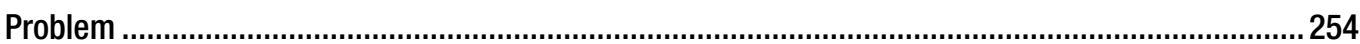

Solution

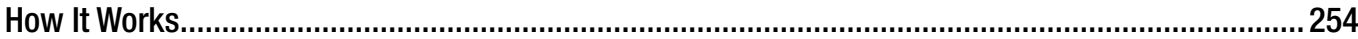

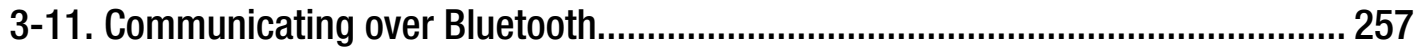

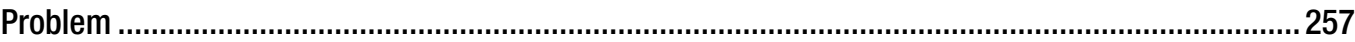

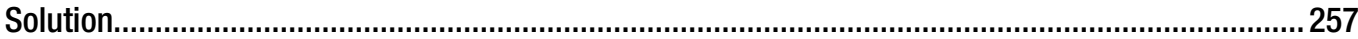

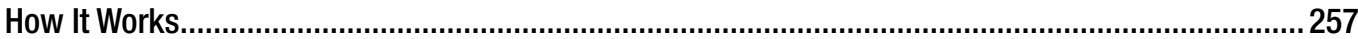

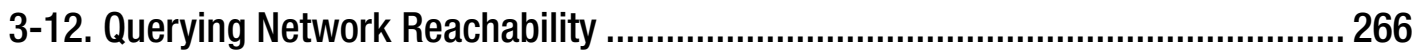

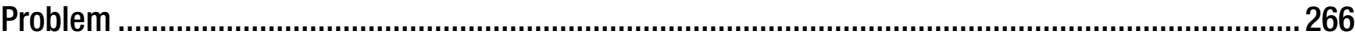

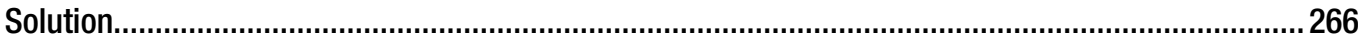

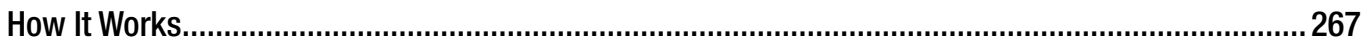

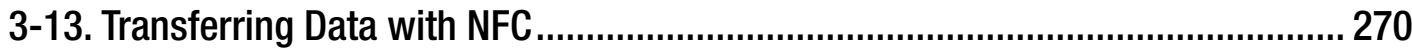

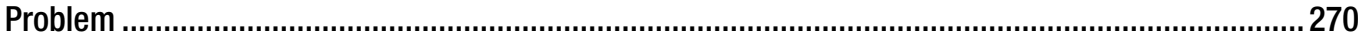

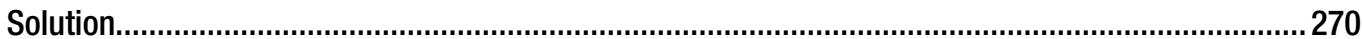

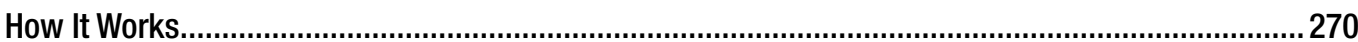

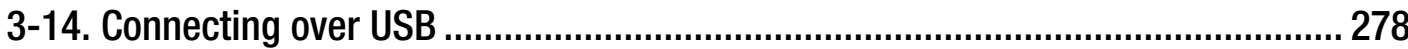

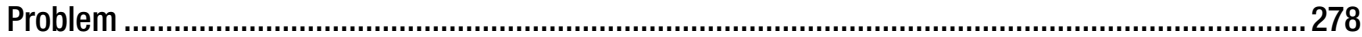

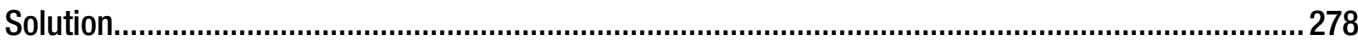

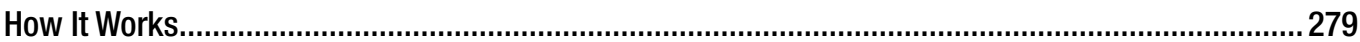

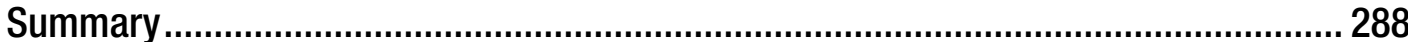

Chapter 4: Interacting with Device Hardware and Media................................. 289

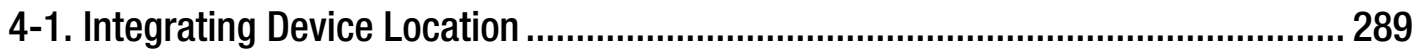

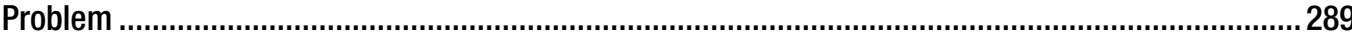

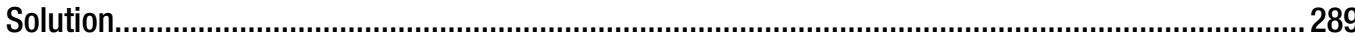

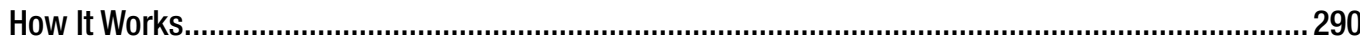




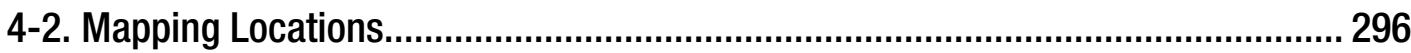

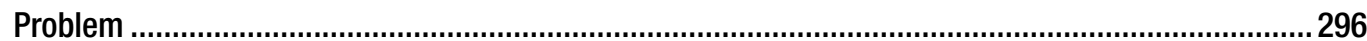

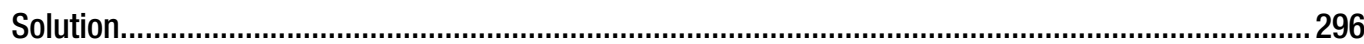

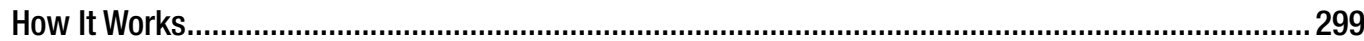

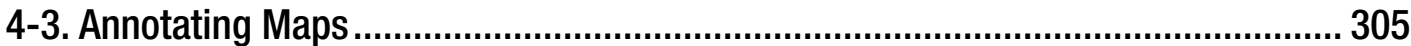

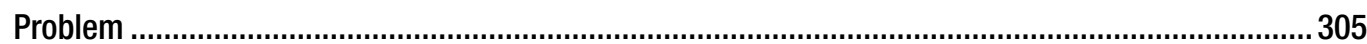

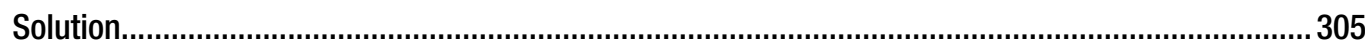

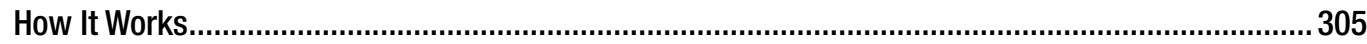

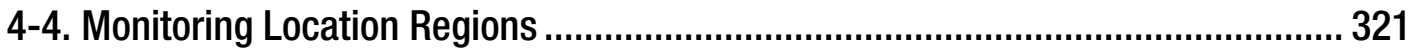

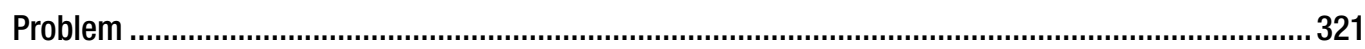

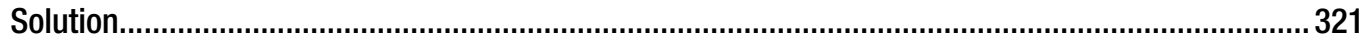

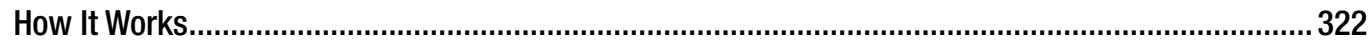

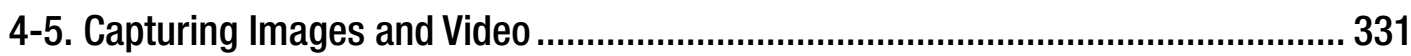

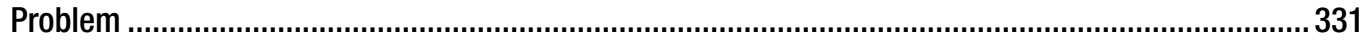

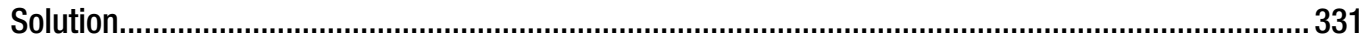

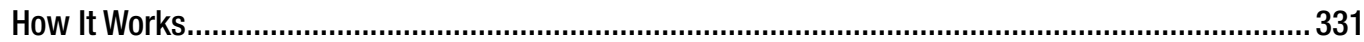

4-6. Making a Custom Camera Overlay...................................................................... 337

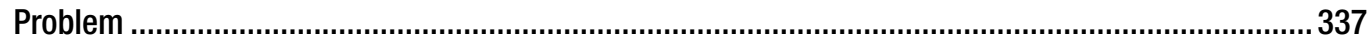

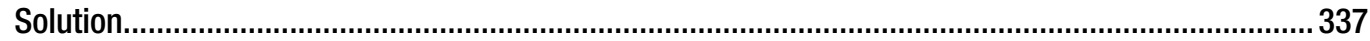

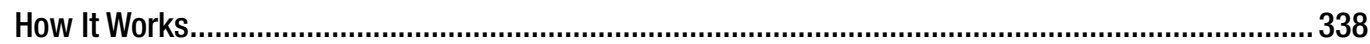

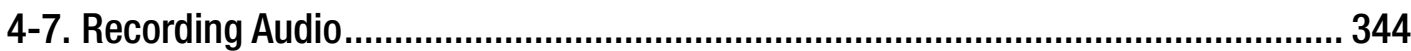

Problem

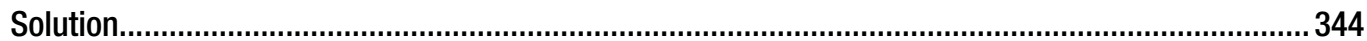

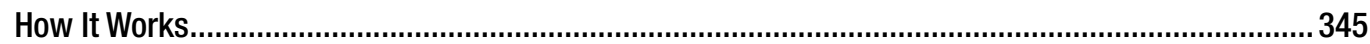

4-8. Capturing Custom Video.................................................................................. 347

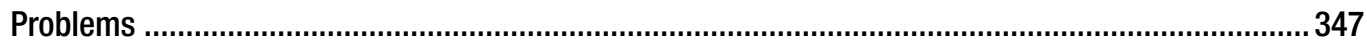

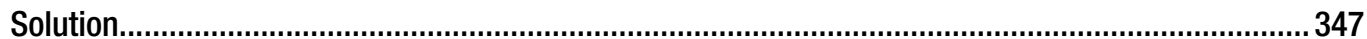

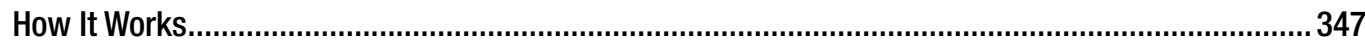

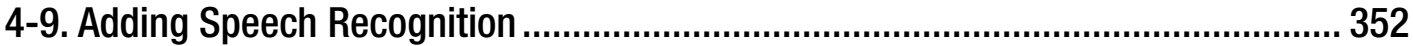

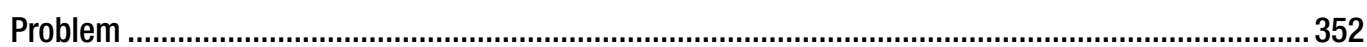

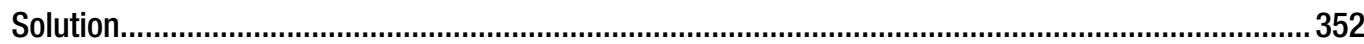

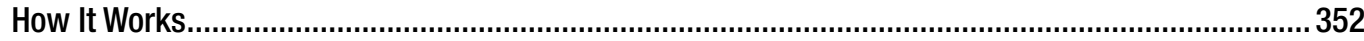




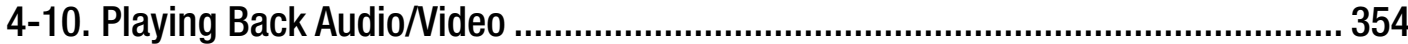

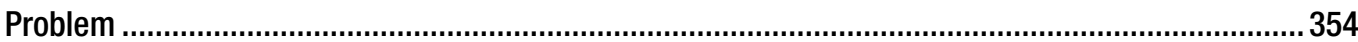

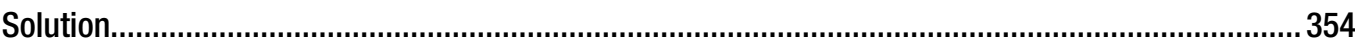

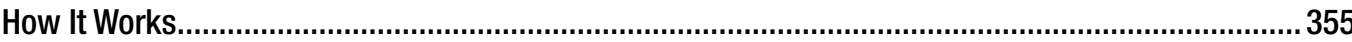

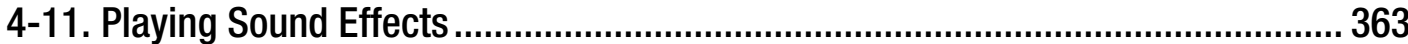

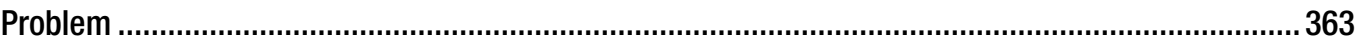

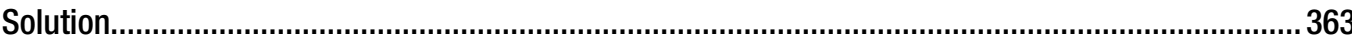

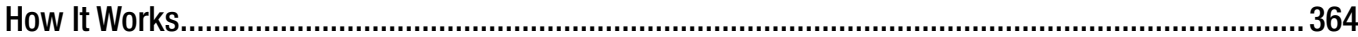

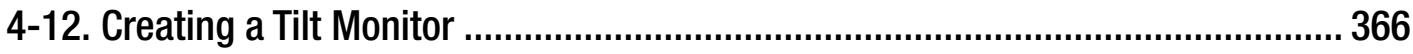

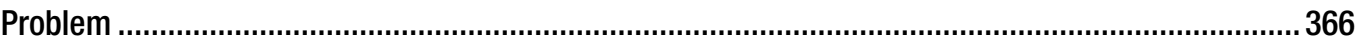

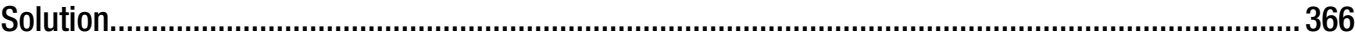

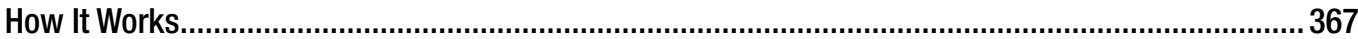

4-13. Monitoring Compass Orientation ................................................................... 371

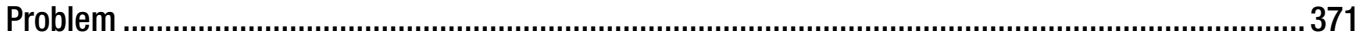

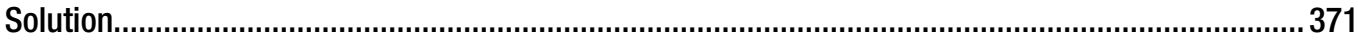

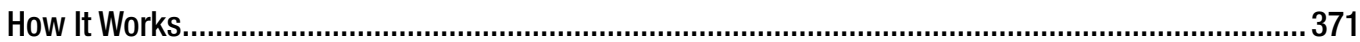

4-14. Retrieving Metadata from Media Content.................................................... 375

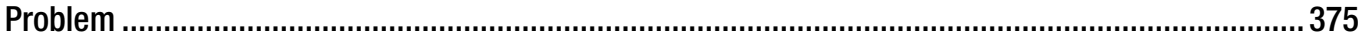

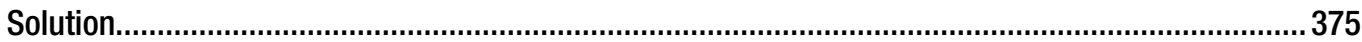

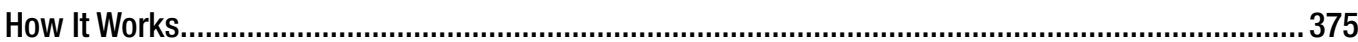

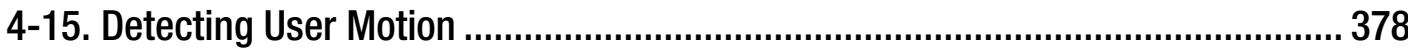

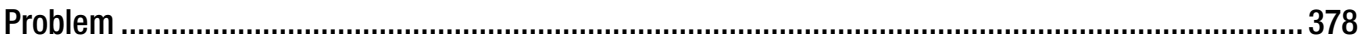

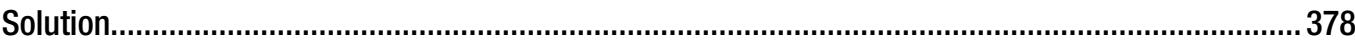

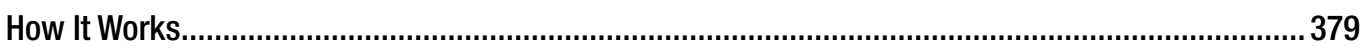

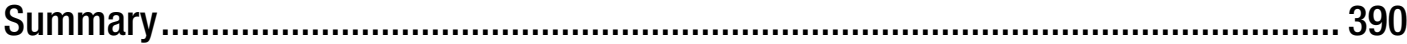

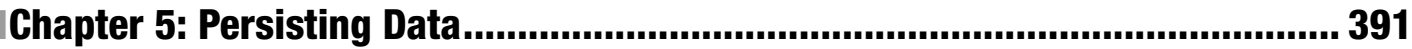

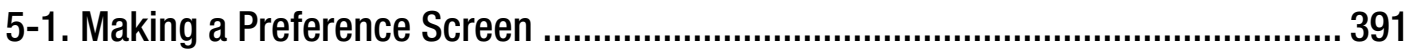

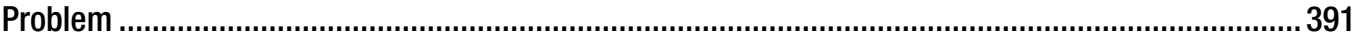

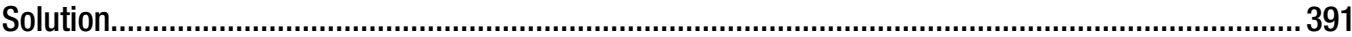

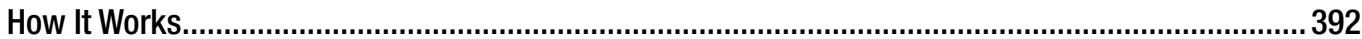




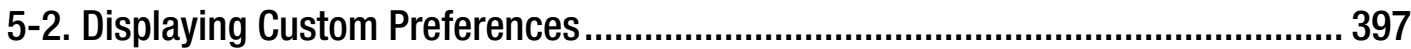

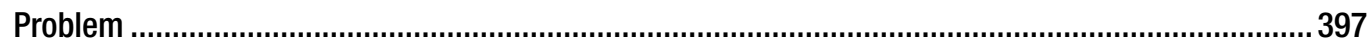

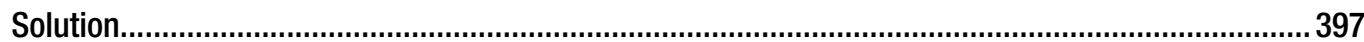

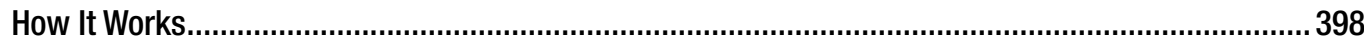

5-3. Persisting Simple Data...................................................................................... 403

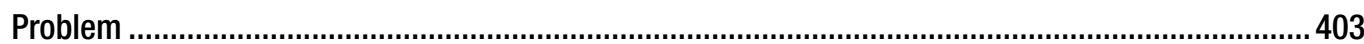

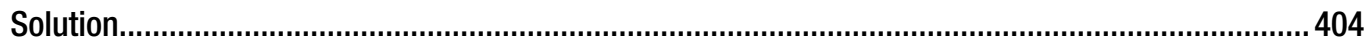

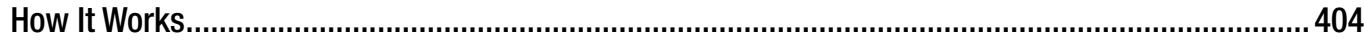

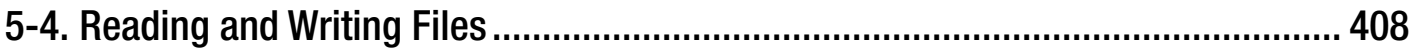

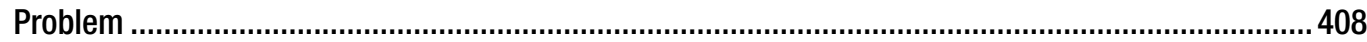

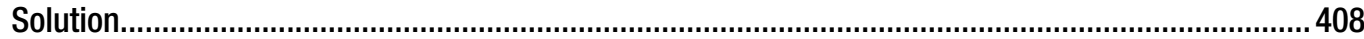

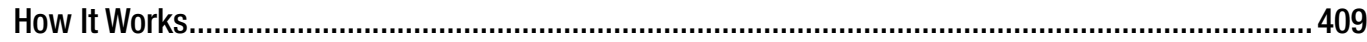

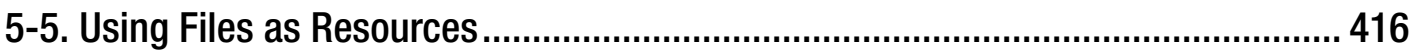

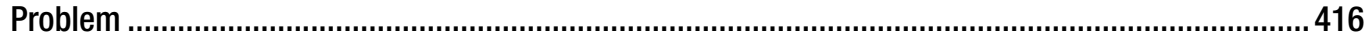

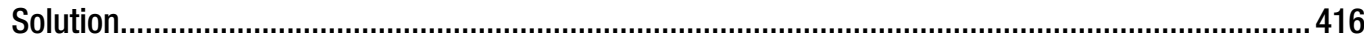

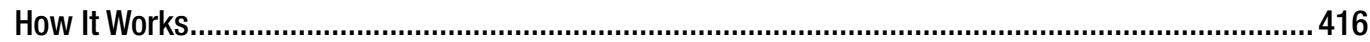

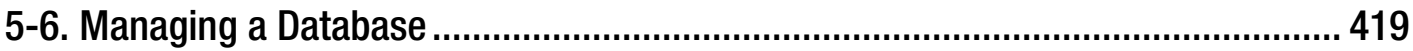

Problem

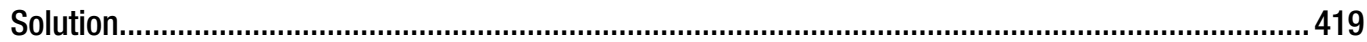

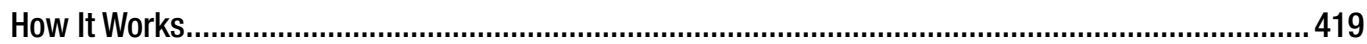

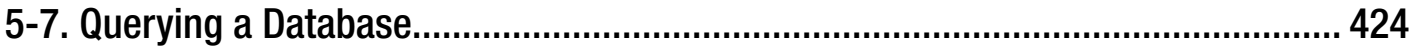

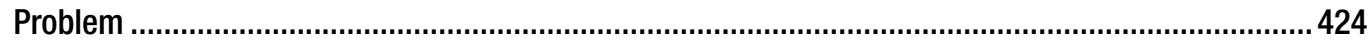

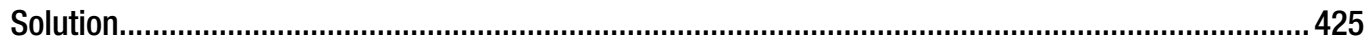

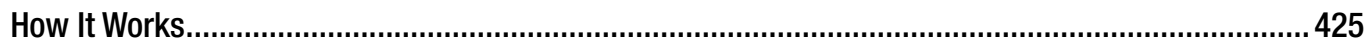

5-8. Backing Up Data ........................................................................................ 426

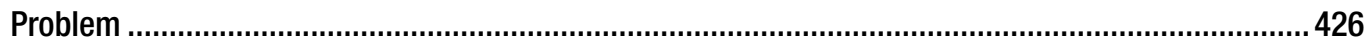

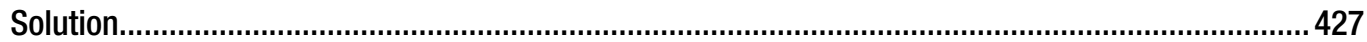

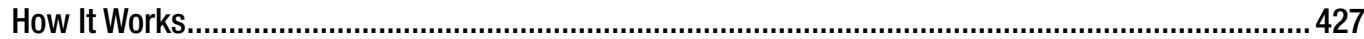




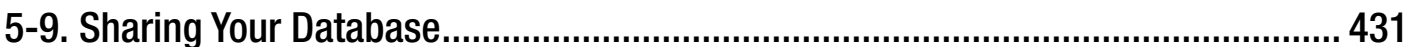

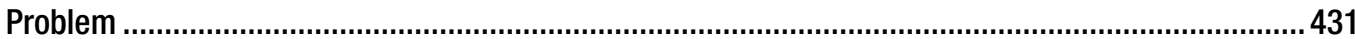

Solution

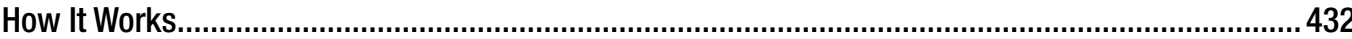

5-10. Sharing Your SharedPreferences .................................................................. 439

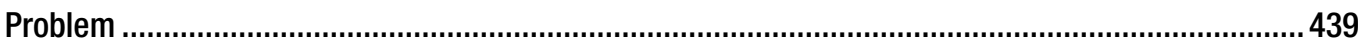

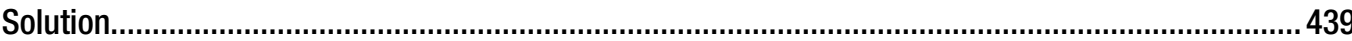

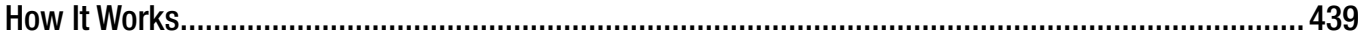

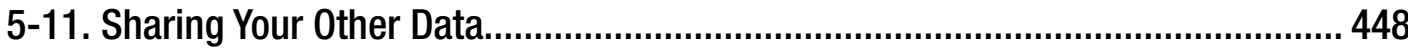

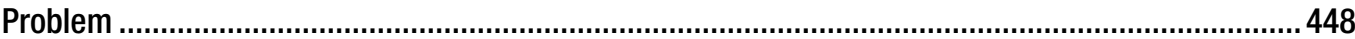

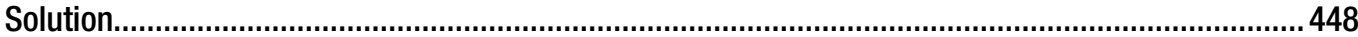

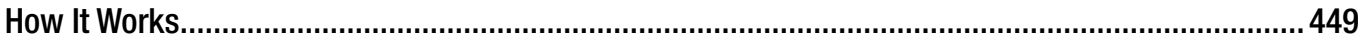

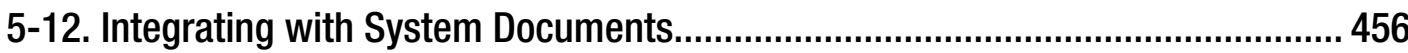

Problem

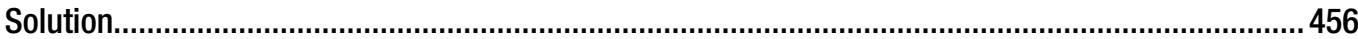

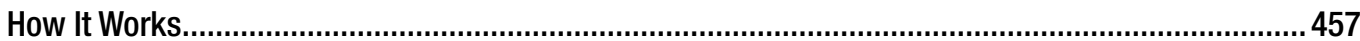

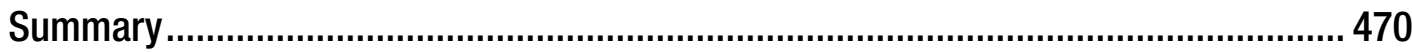

Chapter 6: Interacting with the System........................................................ 471

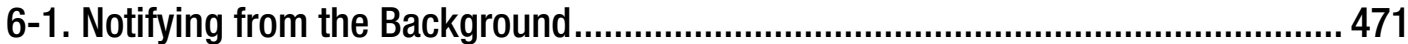

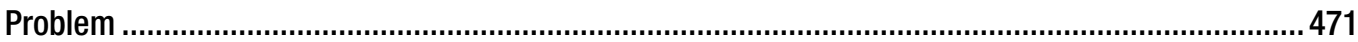

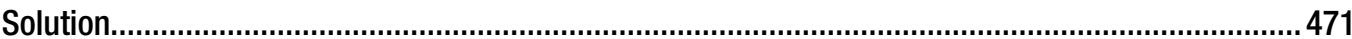

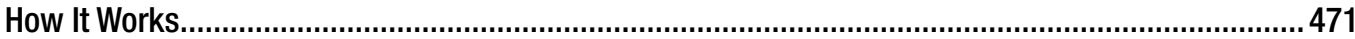

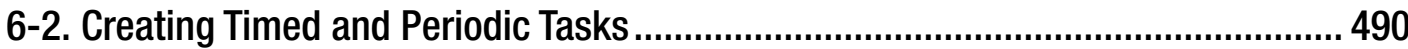

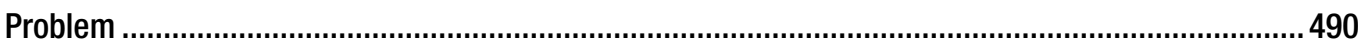

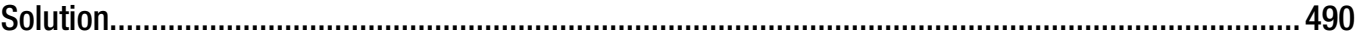

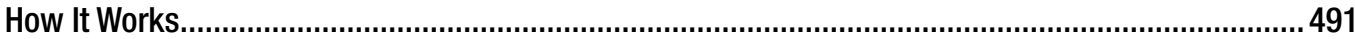

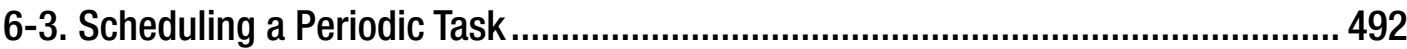

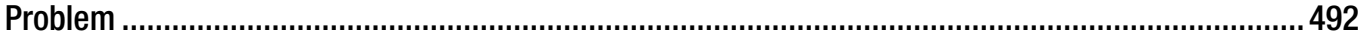

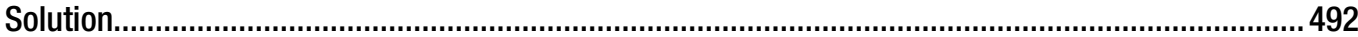

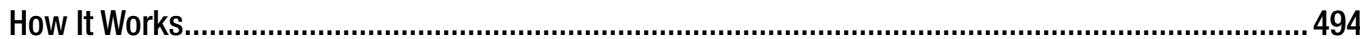




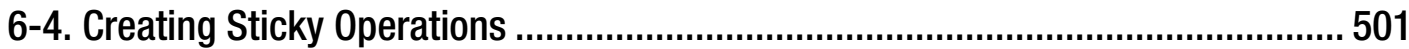

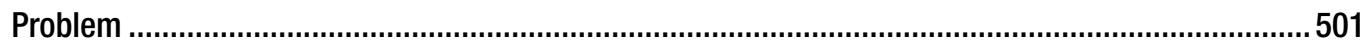

Solution

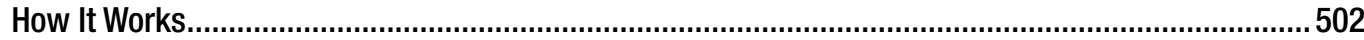

6-5. Running Persistent Background Operations .................................................... 506

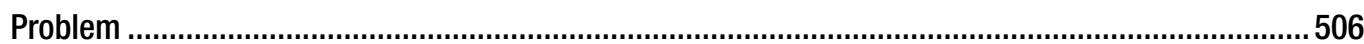

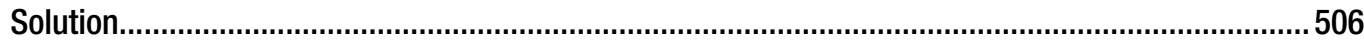

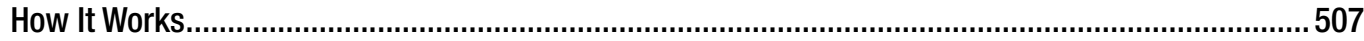

6-6. Launching Other Applications ...................................................................... 513

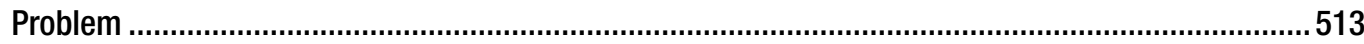

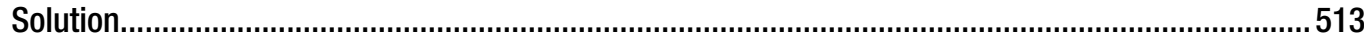

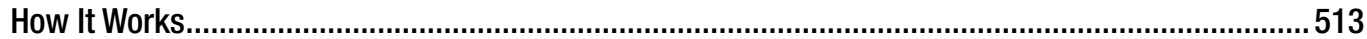

6-7. Launching System Applications.................................................................. 517

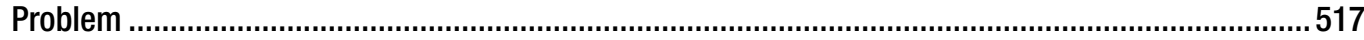

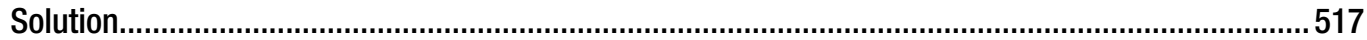

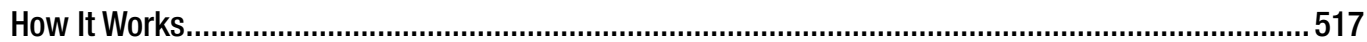

6-8. Letting Other Applications Launch Your Application ......................................... 522

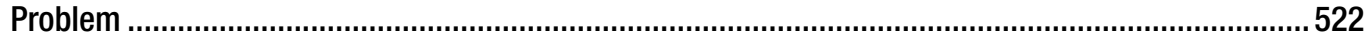

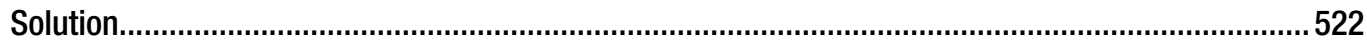

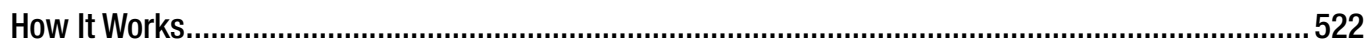

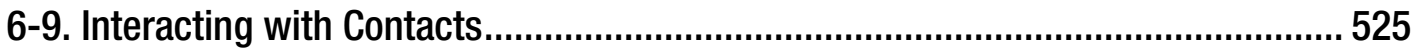

Problem

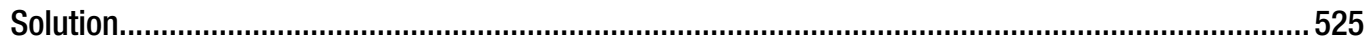

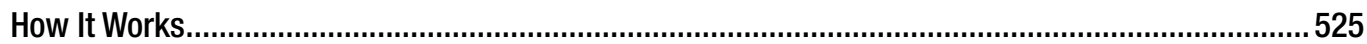

6-10. Reading Device Media and Documents ............................................................. 534

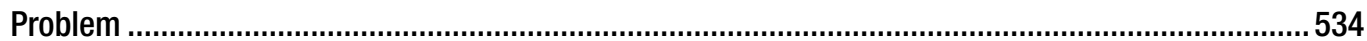

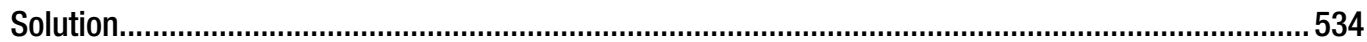

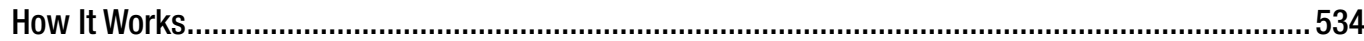

6-11. Saving Device Media and Documents .......................................................... 538

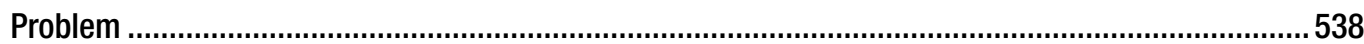

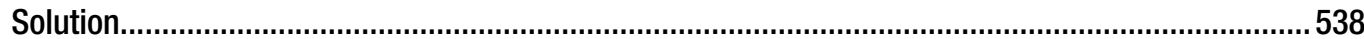

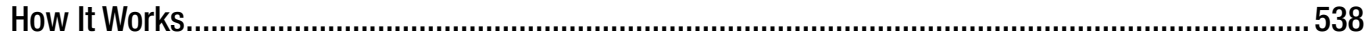




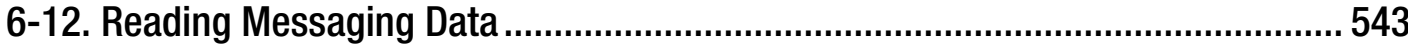

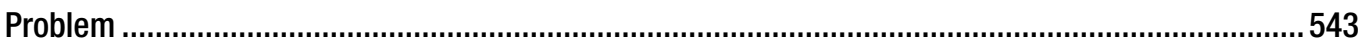

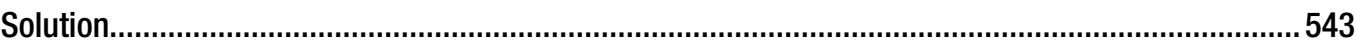

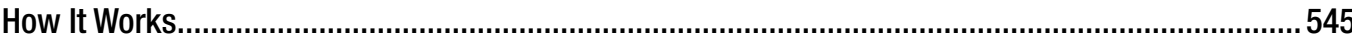

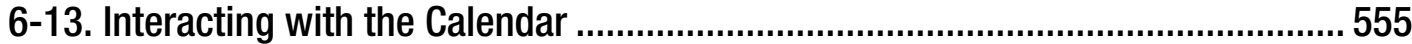

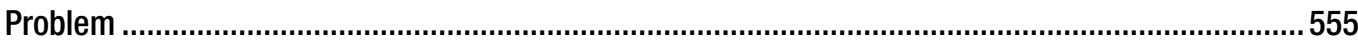

Solution

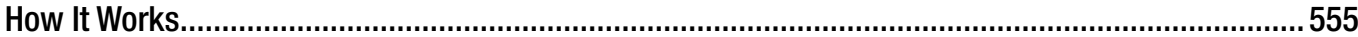

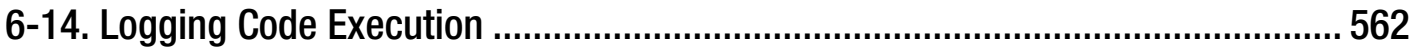

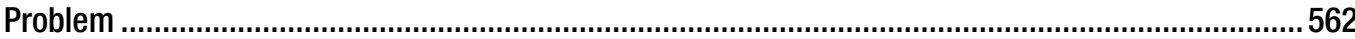

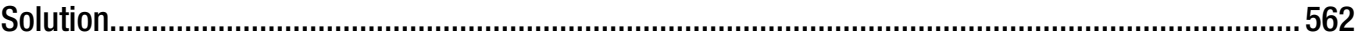

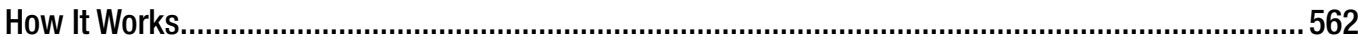

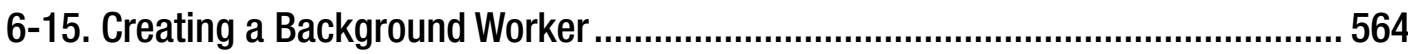

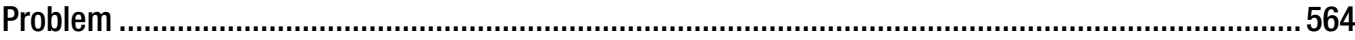

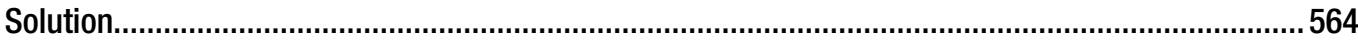

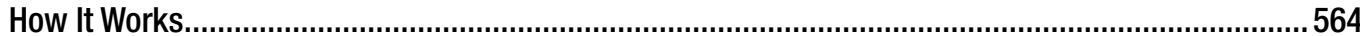

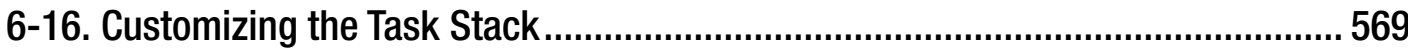

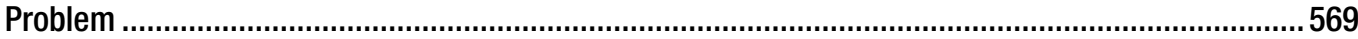

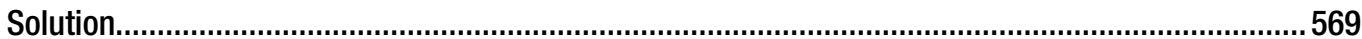

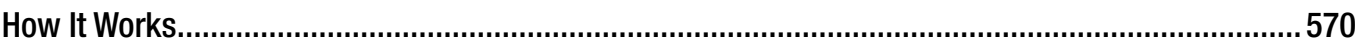

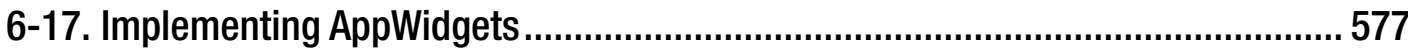

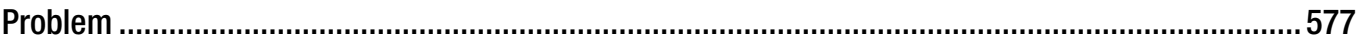

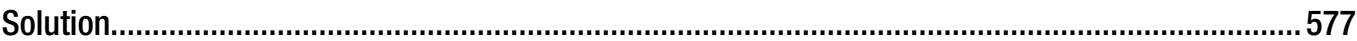

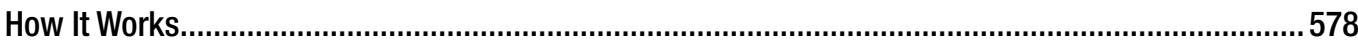

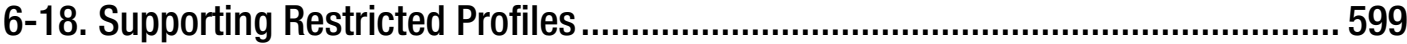

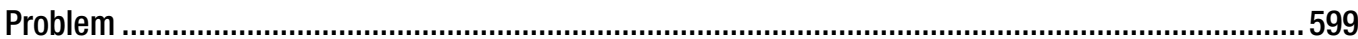

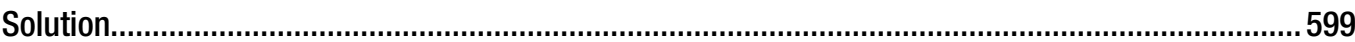

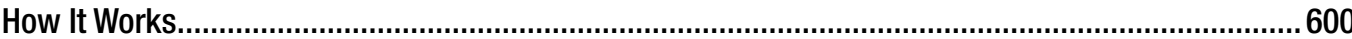

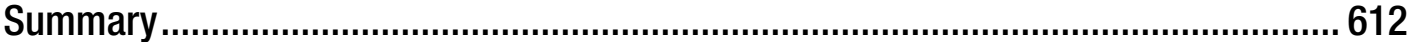


Chapter 7: Graphics and Drawing ................................................................. 613

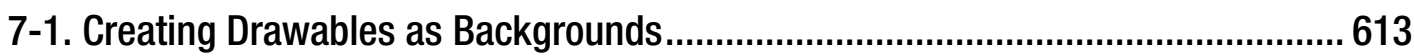

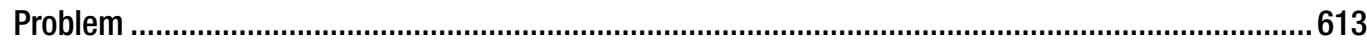

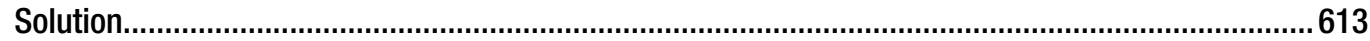

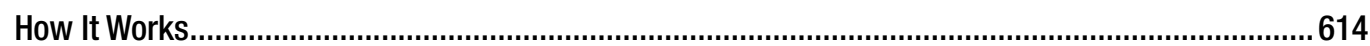

7-2. Creating Custom State Drawables ................................................................... 621

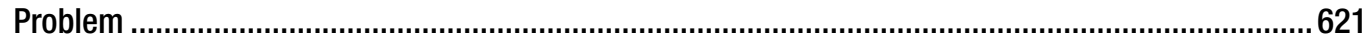

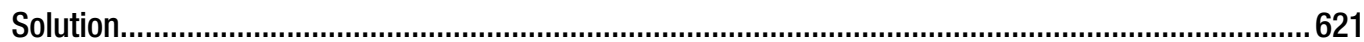

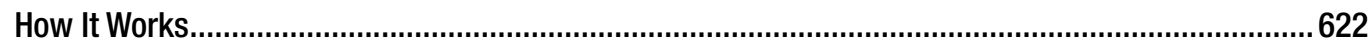

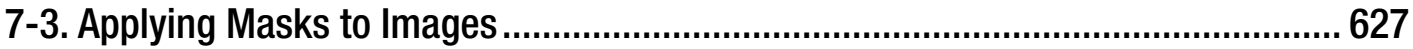

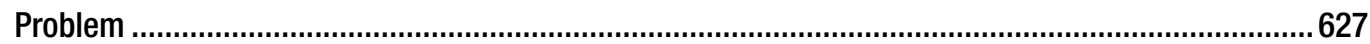

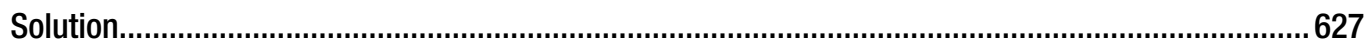

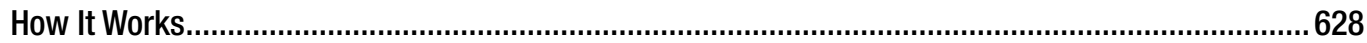

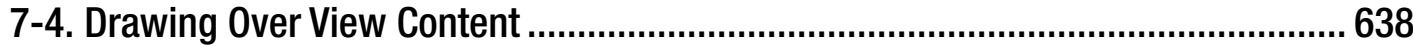

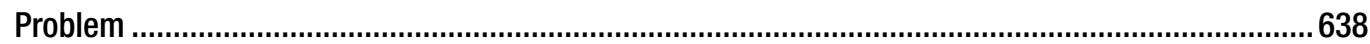

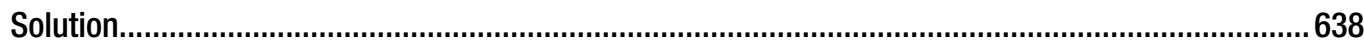

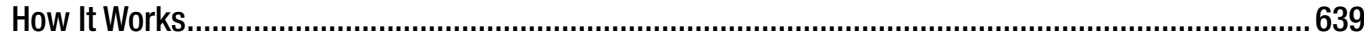

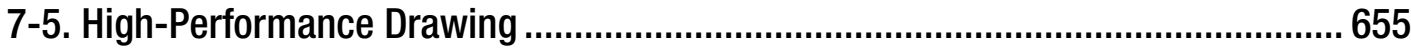

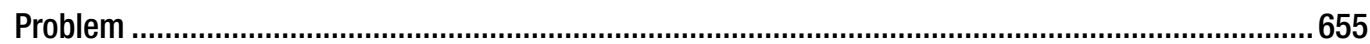

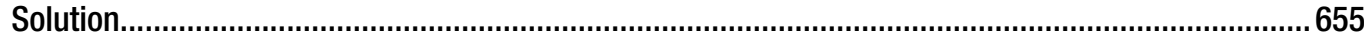

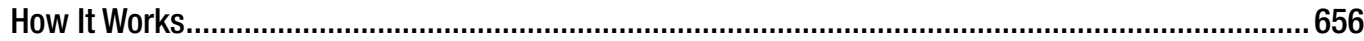

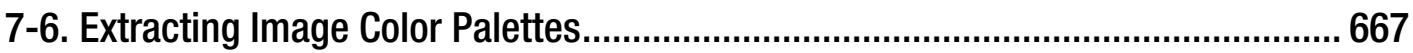

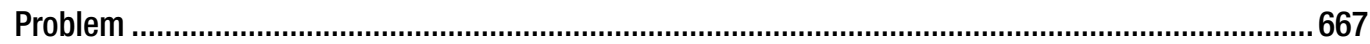

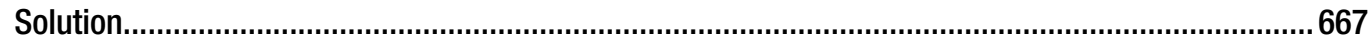

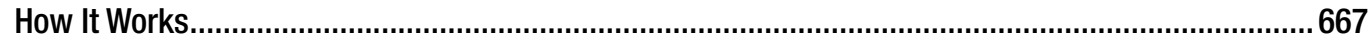

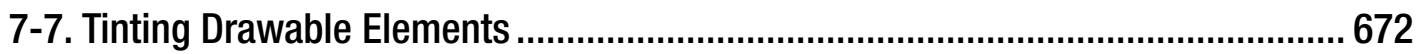

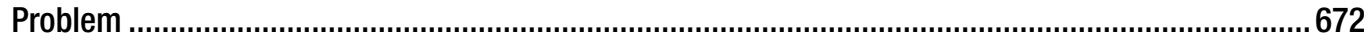

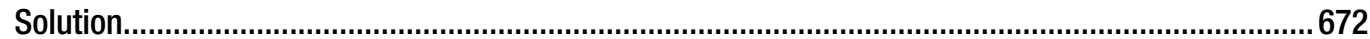

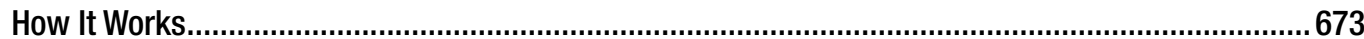




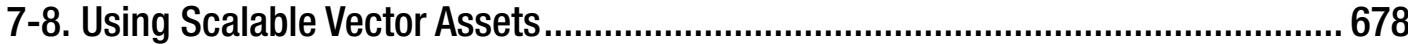

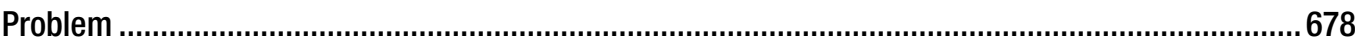

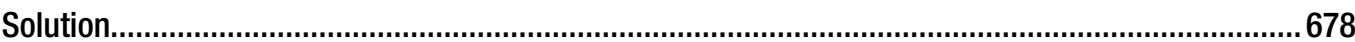

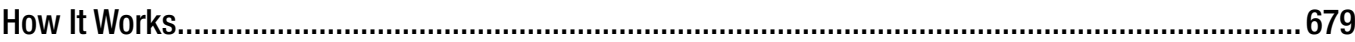

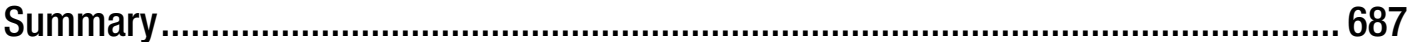

Chapter 8: Working with Android NDK and RenderScript...................................689

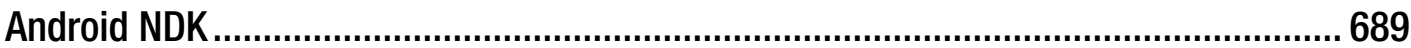

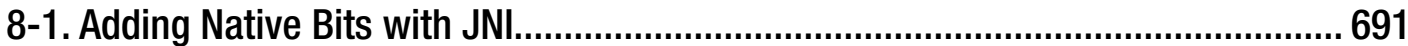

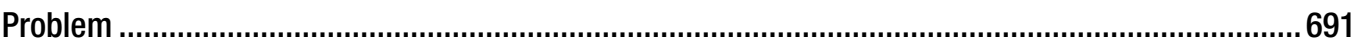

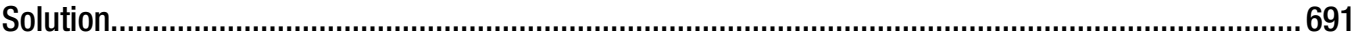

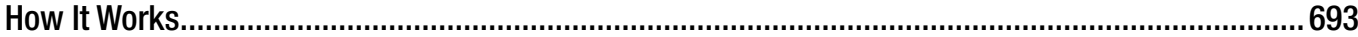

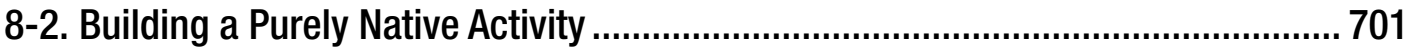

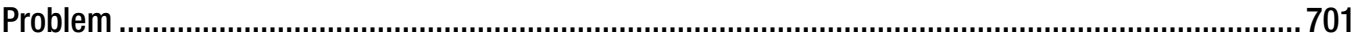

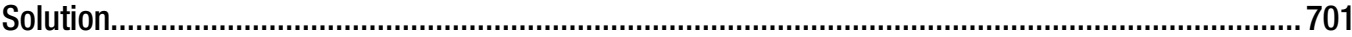

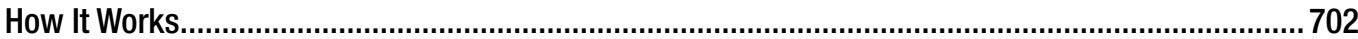

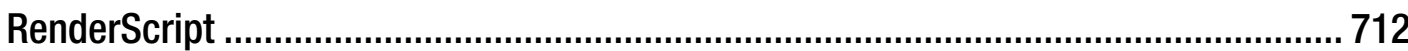

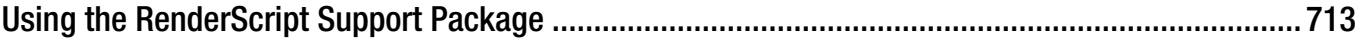

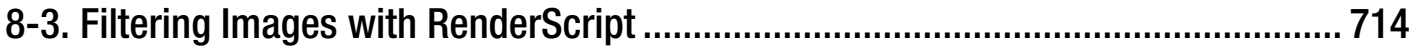

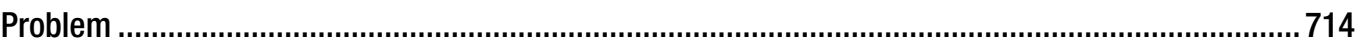

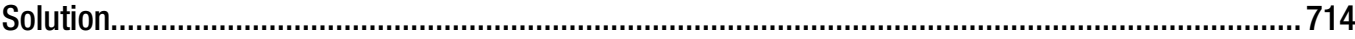

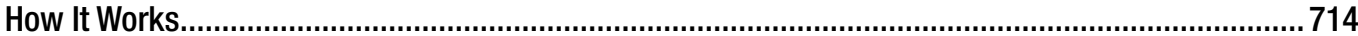

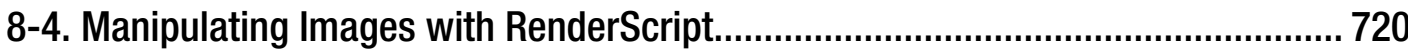

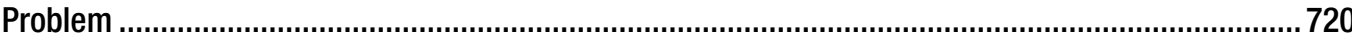

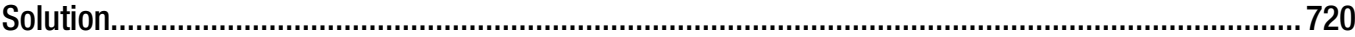

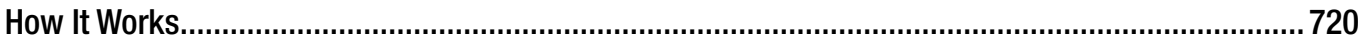

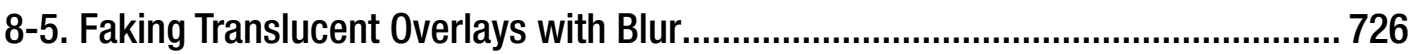

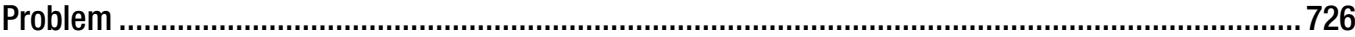

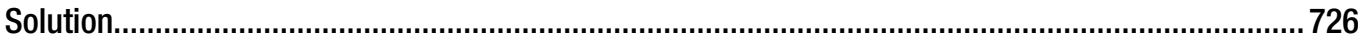

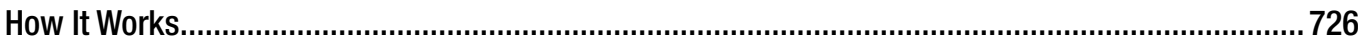

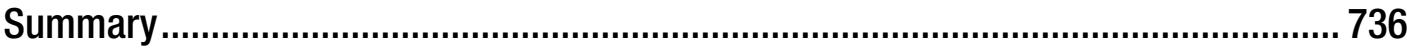





\section{About the Author}

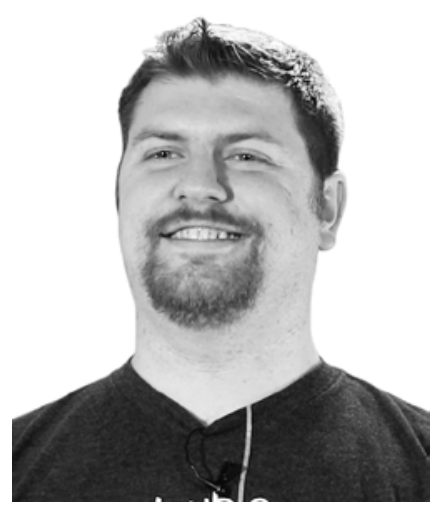

Dave Smith is a professional engineer developing hardware and software for mobile and embedded platforms. Dave's engineering efforts are currently focused full-time on Android development. Since 2009, Dave has worked on developing at all levels of the Android platform, from writing user applications using the software development kit, to building and customizing the Android source code. Dave regularly communicates via his development blog (http://blog.wiresareobsolete.com) and Twitter stream @devunwired. 



\section{About the Technical}

\section{Reviewer}

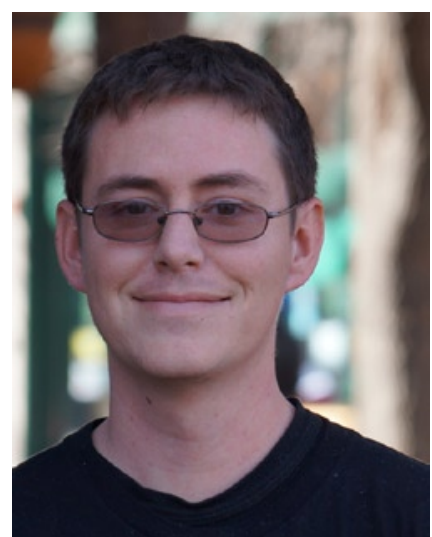

Paul Trebilcox-Ruiz is an Android developer in Boulder, Colorado, and active member in the local Boulder/Denver tech scene. Since moving to Colorado, he has participated in and won multiple hackathons, presented for GDG Denver and has worked on multiple civic coding projects. He currently works on the Android platform at SportsLabs, building and designing applications for university athletics programs across the United States.

Android Recipes is the first book that Paul has contributed to, and an earlier edition of the book was the first Android book that he purchased when learning the platform while obtaining his BS in computer science from California State University, Fresno. 



\section{Acknowledgments}

First and foremost, I would like to thank my wife, Lorie, for her eternal patience and support during the long hours I spent compiling and constructing the materials for this book. Second, I send a huge thank you to the editorial team that Apress brought together to work with me and make the book the best it could possibly be; you guys are the ones who make me look good. Without your time and effort, this project would not even exist. 



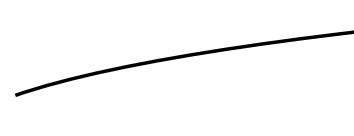

\section{Introduction}

Welcome to the fourth edition of Android Recipes!

If you are reading this book, you probably don't need to be told of the immense opportunity that mobile devices represent for software developers and users. In recent years, Android has become one of the top mobile platforms for device users. This means that you, as a developer, must know how to harness Android so you can stay connected to this market and the potential that it offers. But any new platform brings with it uncertainty about best practices and solutions to common needs and problems.

What we aim to do with Android Recipes is give you the tools to write applications for the Android platform through direct examples targeted at the specific problems you are trying to solve. This book is not a deep dive into the Android SDK, NDK, or any of the other tools. We don't weigh you down with all the details and theory behind the curtain. That's not to say that those details aren't interesting or important. You should take the time to learn them, as they may save you from making future mistakes. However, more often than not, they are simply a distraction when you are just looking for a solution to an immediate problem.

This book is not meant to teach you Java programming or even the building blocks of an Android application. You won't find many basic recipes in this book (such as how to display text with TextView, for instance), as we feel these are tasks easily remembered once learned. Instead, we set out to address tasks that developers, once comfortable with Android, need to do often but find too complex to accomplish with a few lines of code.

Treat Android Recipes as a reference to consult, a resource-filled cookbook that you can always open to find the pragmatic advice you need to get the job done quickly and well. 


\section{What Will You Find in the Book?}

We dive into using the Android SDK to solve real problems. You will learn tricks for effectively creating a user interface that runs well across device boundaries. You will become a master at incorporating the collection of hardware (radios, sensors, and cameras) that makes mobile devices unique platforms. We'll even discuss how to make the system work for you by integrating with the services and applications provided by Google and various device manufacturers.

Performance matters if you want your applications to succeed. Most of the time, this isn't a problem because the Android runtime engines get progressively better at compiling bytecode into the device's native code. However, you might need to leverage the Android NDK to boost performance. Chapter 8 offers you an introduction to the NDK and integrating native code into your application using Java Native Interface (JNI) bindings.

The NDK is a complex technology, which can also reduce your application's portability. Also, while good at increasing performance, the NDK doesn't address multicore processing very well for heavy workloads. Fortunately, Google has eliminated this tedium and simplified the execute-on-multiple-cores task while achieving portability by introducing RenderScript. Chapter 8 introduces you to RenderScript and shows you how to use its compute engine (and automatically leverage CPU cores) to process images.

\section{Keep a Level Eye on the Target}

Throughout the book, you will see that we have marked most recipes with the minimum API level that is required to support them. Most of the recipes in this book are marked API Level 1, meaning that the code used can be run in applications targeting any version of Android since 1.0. However, where necessary, we use APIs introduced in later versions. Pay close attention to the API level marking of each recipe to ensure that you are not using code that doesn't match up with the version of Android your application is targeted to support. 